\title{
Tuning the Matrix Metalloproteinase-1 Degradability of Peptide Amphiphile Nanofibers through Supramolecular Engineering
}

Received 00th January 20xx, Accepted 00th January 20xx

DOI: $10.1039 / \times 0 \times x 00000 x$

\begin{abstract}
Yejiao Shi, ${ }^{a}$ Daniela S. Ferreira, ${ }^{a}$ Jayati Banerjee, ${ }^{a}$ Andrew R. Pickford ${ }^{b}$ and Helena S. Azevedo ${ }^{a}$ *
Matrix metalloproteinases (MMPs) are a family of endopeptidases capable of degrading extracellular matrix (ECM) components. They are known to play crucial roles during the ECM turnover in both physiological and pathological processes. As such, their activities are utilized as biological stimuli to engineer MMP-responsive peptide-based biomaterials such as self-assembled peptide amphiphiles (PAs). Although previous studies have unveiled the role of PAs secondary structure on the mechanical and biological properties of their self-assembled nanostructures, the effect on the degradability of their assemblies by MMP-1 has not been reported. Herein, a series of PAs are designed and synthesized, all comprising the same MMP-1 cleavable domain but with variable structural segments, to decipher the role of PA's secondary structure on the MMP-1 degradability of their assemblies. This study reveals a correlation between the MMP-1 degradation efficiency and the $\beta$-sheet content of the self-assembled PA nanofibers, with the MMP-1 cleavability being significantly reduced in the PA nanofibers with stronger $\beta$-sheet characteristics. These results shed light on the role of supramolecular cohesion in PA assemblies on their hydrolysis by MMP-1 and open up the possibility to control the degradation rate of PA-based nanostructures by MMP-1 through tweaking their molecular sequences.
\end{abstract}

\section{Introduction}

Matrix metalloproteinases (MMPs) comprise a group zincdependent endopeptidases that are responsible for the degradation of extracellular matrix (ECM) components, such as collagens, proteoglycans and glycoproteins. ${ }^{1}$ Their activities are low in normal physiological conditions but increase during the remodelling or repair processes and in inflamed or diseased tissues. ${ }^{2}$ For example, during the remodelling stage of wound healing, the upregulation of MMP-1 is crucial, since MMP-1 not only breaks down the proteins in the provisional ECM barrier to permit cell migration, but also mediates the balance between ECM synthesis and degradation. ${ }^{3}$ Other MMPs, such as MMP-2 and MMP-9, are found overexpressed in breast, colorectal, prostate and many other types of cancers, and are recognized as the most prominent proteinases associated with tumour progression and metastasis. ${ }^{4,5}$ These abnormal MMP activities have been considered as attractive biological triggers to design responsive biomaterials with the capacity to exert their functions specifically and selectively. ${ }^{6-8}$

Among the building blocks used for creating biomaterials, selfassembling peptide amphiphiles (PAs) developed by the Stupp laboratory have received considerable attention over the past decades due to their advantageous properties, such as

\footnotetext{
a. School of Engineering and Materials Science \& Institute of Bioengineering, Queen Mary, University of London, E1 4NS, UK, E-mail: h.azevedo@qmul.ac.uk

b. Centre for Enzyme Innovation \& School of Biological Sciences, University of Portsmouth, PO1 2DY, UK

†Electronic Supplementary Information (ESI) available: [Supporting Figures]. See DOI: $10.1039 / x 0 x x 00000 x$
}

biocompatibility, biodegradability, as well as design versatility over both the structure and function of their assemblies. ${ }^{9}$ Typically, a PA molecule consists of a hydrophobic alkyl tail covalently attached to a tuneable peptide segment. Through rational design, a wide variety of nanostructures, such as fibres, ribbons, belts and micelles, were observed in PA assemblies. These nanostructures have been explored in diverse medical applications, ranging from tissue engineering, regenerative medicine, immune modulation to drug delivery and disease diagnostics. ${ }^{9-11}$

The incorporation of different MMP-cleavable peptide sequences into self-assembling PAs, have enabled the design of nanostructures with properties tailored for specific medical purposes. For instance, nanostructures assembled from PAs containing MMP substrates were explored to mimic the ECM degradation process for tissue engineering applications, a concept first proposed by Hubbell using polyethylene glycol (PEG) hydrogels crosslinked with MMP-sensitive peptides. ${ }^{12}$ Based on this concept, Hartgerink's group developed selfassembled PA nanofiber networks, which could undergo cellmediated degradation with the incorporation of MMP-2 cleavable sites into the PA molecules. ${ }^{13,}{ }^{14}$ Inspired by these pioneering studies, our group engineered self-assembling membranes made of hyaluronic acid and MMP-1 substrate containing PAs. ${ }^{15}$ These membranes were shown to respond to the hydrolytic activities of both hyaluronidase and MMP-1, stimulate MMP-1 secretion by fibroblasts and interfere with the collagen deposition, which highlighted the ability of modulating certain cellular activities through ECM engineering. ${ }^{15}$ Moreover, nanostructures assembled from MMP substrate containing PAs were also explored to respond to the activities of MMPs 
overexpressed at the tumour environment for cancer therapy. Through complexation of the anticancer drug cisplatin with the free carboxylates in a PA molecule, a cisplatin delivery system was developed based on a MMP-2 sensitive PA gel, which could release cisplatin when exposed to MMP-2. ${ }^{16} \mathrm{MMP}-7$ induced hydrogelation of PAs was also described. ${ }^{17}$ The designed PA was used as a hydrogelator precursor, which presented a sol-gel phase transition upon the removal of a gelation-preventing moiety in its sequence by MMP-7 proteolysis. ${ }^{17}$ The MMP-7 triggered hydrogelation of the PA was further proved to selectively initiate cancer cell death via exerting vital stress on cancer cells. ${ }^{18}$ A proof-of-concept design of MMP-2 sensitive PA nanostructures with potential as tumour targeted intracellular delivery vehicles was also reported by our group. ${ }^{19}$ These nanostructures were shown to selectively expose a cellpenetrating peptide at their surface and undergo fibre-tomicelle morphological transition upon degradation by MMP$2 .{ }^{19}$

These studies highlight the interest in exploiting MMP activities to induce desirable functions in self-assembling PAbased biomaterials by selective degradation. To achieve controlled biological outcomes with these biomaterials, either promoting cell migration and growth, or regulating drug release, it is critical to optimize their degradation rate. ${ }^{20}$ Earlier studies by Hubbell's group have identified candidate linear peptides for optimized degradation by MMP-1 and MMP-2, which were further used to enhance the degradation of PEG hydrogels and allow increased cellular infiltration for potentially more robust healing in vivo. ${ }^{21,} 22$ Several attempts were exploited to control the degradability of self-assembling peptide nanofibrous hydrogels by MMP enzymes mainly through the peptide primary structure (e.g. sequence modification of MMP substrates). ${ }^{23}, 24$ Recently, Ulijn and coworkers reported a peptide design approach to tune the degradation kinetics of supramolecular peptide nanostructures by MMP-9. ${ }^{25}$ Peptides were designed with systematically modified amino acid sequences to control overall charge and order in the MMP-9 substrate region. As result of this systematic design, nanostructures with different secondary structures and morphologies (nanofibers, elongated worm-like micelles and spherical micelles) were formed by self-assembly, not being possible to isolate the effect of nanostructure morphology on MMP cleavability. In contrast, PA molecules are known to self-assemble into defined nanofiber structures whose rigidity and biological properties were shown to be regulated through modulation of their secondary structure. ${ }^{26-29}$ However, the effect of PA's secondary structure on their degradability by relevant proteases has not been investigated in detail. MMP-1 is a member of the collagenase family ${ }^{30}$ that includes MMP-8 and MMP-13, and has the ability to cleave interstitial collagens (and, to a much lesser extent, denatured collagen which is also known as gelatin) 31,32 leading to unwinding of the triple-helical structure and leaving the now unstructured substrate highly susceptible to gelatinases, such as MMP-2 and MMP-9. The ability of an MMP collagenase to breakdown triple helical collagen relies on the binding of an $\mathrm{S} 10^{\prime}$ "exosite" within the enzyme's hemopexin domain to a region $C$ - terminal to the substrate's scissile bonds. ${ }^{33}$ This specific interaction is believed to locally unwind the triple helix secondary structure allowing a single $\alpha$-chain to enter the active site cleft of the catalytic domain which is too narrow to accommodate all three $\alpha$-chains simultaneously. ${ }^{34}$ Herein, we hypothesize that the degradability of PA nanofibers by MMP-1 can be tuned by controlling their internal supramolecular cohesion. To test this hypothesis, we performed a detailed study to elucidate the relationship between the secondary structure of self-assembled PA nanostructures and their MMP1 degradability.

\section{Results and discussion}

The specificity of MMP-1 for its substrates, either proteins or peptides, is typically influenced by the substrate sequences and their local secondary and tertiary structure. ${ }^{35}$ The regions containing protease substrates are expected to be flexible to facilitate access by the enzyme active site. The flexibility can be affected by the local secondary structure, which is determined by the primary sequence. The use of synthetic triple-helical peptides in previous studies has provided insights into the collagen degradation by MMPs, ${ }^{36}$ but the effect of hydrogen bonding network around the cleavage site and molecular packing has not been investigated in detail. In this study, we have selected PAs as a model self-assembling system as their self-assembly behaviour has been well established and enables systematic variations in their structural domains.

\section{Design of MMP-1 Substrate Containing PAs with Variable Secondary Structures}

To verify our hypothesis that the molecular structure of PAs can be manipulated to control the degradation efficiency of their assemblies by MMP-1, a series of peptide molecules, all containing the MMP-1 substrate, but with the potential to adopting different secondary structures, were designed. As summarized in Table 1, all the peptide molecules were designed based on an octapeptide MMP-1 substrate (GPQG $\downarrow$ IWGQ), which was reported to be cleaved by MMP-1 at the site between glycine (G) and isoleucine (I). ${ }^{21,37}$ To ensure good solubility of all the designed peptide molecules in water, three charged lysine $(\mathrm{K})$ residues were added to the $C$-terminus of the

Table. 1 Sequence and critical aggregation concentration (CAC) of the designed PS and PAs containing the MMP-1 cleavable peptide sequence (orange: hydrophobic alkyl tail; red: $\beta$-sheet or $\alpha$-helix promoting peptide segment; green: MMP-1 cleavable peptide domain; blue: charged peptide headgroup).

\begin{tabular}{|c|c|c|}
\hline \# & Sequence & $\begin{array}{c}\text { CAC } \\
(\mathrm{mM})\end{array}$ \\
\hline PS & $\mathrm{CH}_{3} \mathrm{CONH}-\mathrm{GPQGIWGQKKK-CONH}{ }_{2}$ & 2 \\
\hline PA1 & $\mathrm{C}_{15} \mathrm{H}_{31} \mathrm{CONH}-\mathrm{GPQGIWGQKKK-CONH}$ & 2 \\
\hline PA2 & $\mathrm{C}_{15} \mathrm{H}_{31} \mathrm{CONH}-\mathrm{AAAAAAGPQGIWGQKKK}-\mathrm{CONH}_{2}$ & 0.04 \\
\hline PA3 & $\mathrm{C}_{15} \mathrm{H}_{31} \mathrm{CONH}-\mathrm{VVVAAAGPQGIWGQKKK-CONH}{ }_{2}$ & 0.004 \\
\hline PA4 & $\mathrm{C}_{15} \mathrm{H}_{31} \mathrm{CONH}-\mathrm{GPQGIWGQVVVAAAKKK-CONH} 2$ & 0.06 \\
\hline
\end{tabular}



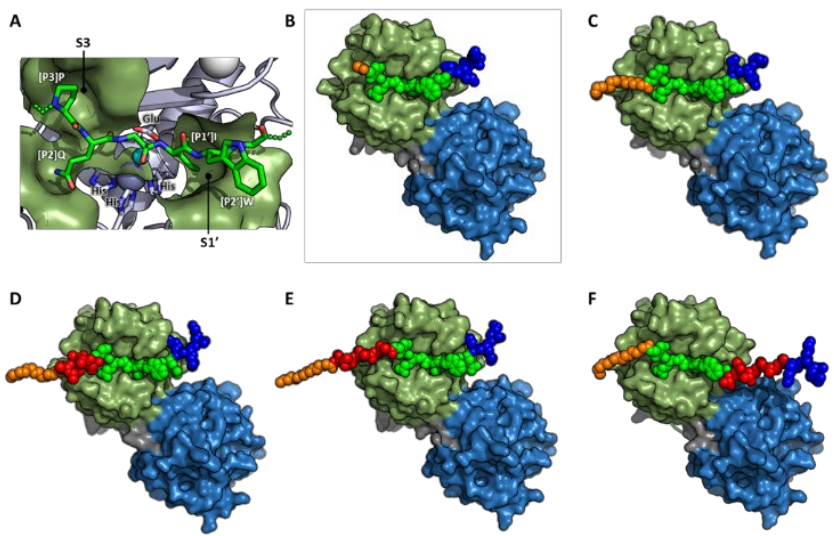

Fig. 1 Models of peptide monomers bound into the catalytic cleft of MMP-1: model of the pre-hydrolysis, binary complex formed between the octapeptide GPQG $\downarrow$ IWGQ (where $\downarrow$ indicates the scissile peptide bond) and the catalytic domain of MMP-1 (A). The catalytic zinc ion (grey sphere), which is coordinated by three histidine (His) residues, polarizes the carbonyl group of the scissile peptide, and coordinates the water molecule (cyan sphere) that will initiate hydrolysis by nucleophilic attack of the carbonyl carbon (indicated by an asterisk). Hydrogen bonding to the neighbouring glutamate (Glu) makes the water a stronger nucleophile. The recognition sub-sites (S3 and S1') that principally determine cleavage specificity are annotated and illustrated with pale green surfaces; Modelled complexes of MMP-1 (in its compact state) bound to PS, PA1, PA2, PA3 and PA4, respectively (B-F). In each image, the MMP-1 surface is depicted with the catalytic domain, hemopexin domain and inter-domain linker coloured pale green, pale blue and grey, respectively. Each peptide is shown space-filled, with a colour scheme identical to that in Table 1.

octapeptide sequence. As for the $N$-terminus, several modifications were implemented. A peptide substrate (PS) was designed where the $N$-terminal amine was acetylated to eliminate the effect of additional charge at the $\mathrm{N}$-terminus, while PA1 was capped with palmitic acid. The hydrophobic effect imparted by the 16-carbon alkyl tail is expected to drive the self-assembly of PA1 in aqueous solution by collapse of the hydrophobic tail in the core and presenting the lysine headgroup on the surface of the assemblies. To design PAs with variable secondary structure, a segment of six amino acids with different propensity of intermolecular hydrogen bonds was incorporated adjacent to the hydrophobic alkyl tail. In PA2, an $\alpha$-helix promoting peptide sequence, composed of alanine residues (AAAAAA), ${ }^{38,39}$ was incorporated next to the alkyl tail, while a well-known $\beta$-sheet forming peptide segment (VVVAAA) was used to generate PA3, which has been reported to form $\beta$ sheet nanofibers in our previous study. ${ }^{15}$ PA4 was designed by swapping the position of the $\beta$-sheet forming and MMP-1 cleavable peptide domains, with the assumption that PA4 may adopt a relatively weak or even disrupted $\beta$-sheet secondary structure as the $\beta$-sheet forming peptide segment is located away from the hydrophobic alkyl tail. ${ }^{40}$ Keeping the overall charge unchanged, and solely altering the secondary structure promoting segment as well as the location of the MMP-1 cleavable domain within the PA molecules, allow us to probe the effect of the internal packing of PA assemblies on the ability of MMP-1 to hydrolyse the peptide monomers at the G-I peptide bond.

\section{In Silico Validation of the Designed PAs}

To assess the theoretical potential of each designed peptide molecule to bind to the active site of MMP-1 when in a monomeric state, in silico modelling of the pre-hydrolysis (Michaelis) complex was performed. This assessment is particularly important for any peptide sequence which promotes, in the vicinity of the scissile bond, a defined conformation since the catalytic cleft can only accommodate substrates that are locally unstructured. ${ }^{34}$ The success of such analyses are dependent upon an accurate portrayal of specific enzyme-substrate interactions within the active site. Therefore, the likely conformation of the octapeptide substrate was established within the MMP-1 catalytic cleft by reference to crystal structures of the catalytic domain of MMP-8, which is highly homologous with that of MMP-1 (Fig. S1At), in complex with inhibitors that occupy either the $\mathrm{S1}^{\prime}$ pocket $^{41}$ or the S3 pocket $^{42}$ (Fig. S1B, C+); together these pockets are the chief determinants of MMP-1 substrate specificity. ${ }^{43}$ Those prior analyses, when combined with the crystal structure of MMP$1,{ }^{44}$ provided sufficient structural information for reliably positioning the [P3]Pro and [P1']lle residue sidechains into their respectively sub-sites, and for establishing a backbone conformation that places the scissile peptide bond in close proximity to the zinc ion and Glu residue that are both essential for MMP catalytic activity (Fig. 1A). Unfortunately, at 3 residues long, the MMP-8 inhibitors used in previous crystal structures are too short to provide conformational information for the first or the last amino acids of the octapeptide. However, since these sites (P4 and P4') confer no detectable substrate specificity in MMP $-1,43$ there are unlikely to be specific or extensive contacts between the catalytic domain and [P4]Gly or [P4']GIn of the octapeptide. Hence, the conformation of these residues were not constrained in subsequent modelling studies.

The octapeptide extends sufficiently far from the catalytic cleft that the tri-lysine $C$-terminal imparts no appreciable steric hindrance on binding for either PS or any of the PAs (Fig. 1B-F). As expected, due to its unbranched structure, the palmitic acid tail at the $N$-terminal of each PA substrate is also readily accommodated into each model of MMP-1 in complex with a monomeric peptide. However, it is apparent that, particularly for PA1 and PA4, the close proximity of the fatty acid chain to the enzyme may preclude binding when these peptides successfully assemble into nanofibers or any other nanostructures (Fig. 1C-F). For PA2-PA4, the secondary structure elements could also be introduced without encountering any steric hindrance with either domain of MMP1 (Fig. 1D-F), and without straining the established conformation of the octapeptide substrate within the active site, as judged by the overall potential energy of the system, and the lack of unrealistically-close non-bonded contacts; it is particularly notable that the AAAAAA segment in PA2 has the potential to form an $\alpha$-helix (which is comparatively bulky) without hindering binding of the more-extended scissile sequence into the MMP-1 catalytic cleft. Having established that the $N$-terminal palmitic acid adduct, the $C$-terminal tri- 

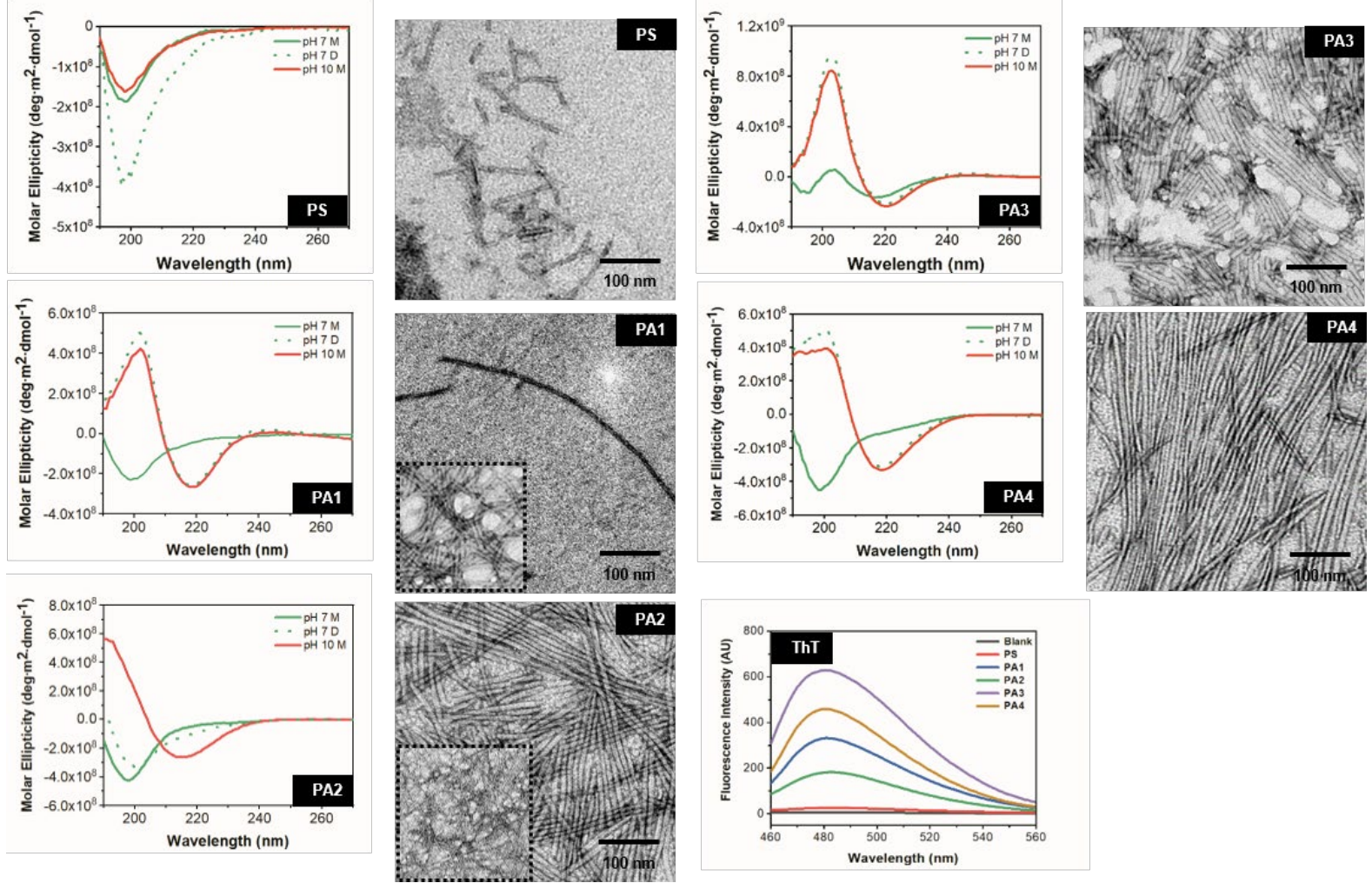

Fig. 2 Structure characterization and self-assembly of the designed PS and PAs in water: CD spectra of PS, PA1, PA2, PA3 and PA4 in the monomeric state (M, solid line, prepared at concentration < CAC) and their diluted aggregates (D, dashed line, prepared at concentration > CAC first and then diluted to the same concentration as in the monomeric state) at $\mathrm{pH} 7$ and $\mathrm{pH} 10$ displaying different secondary structures; representative TEM images of PS, PA1, PA2, PA3 and PA4 showing various fibrous nanostructures at $\mathrm{pH}$, and also $\mathrm{pH} 10$ (inserted images with dash line border); fluorescence of ThT in the presence of PS, PA1, PA2, PA3 and PA4 nanofibers in water at $\mathrm{pH} 7$ showing differences in the $\beta$-sheet content within the various nanofibers.

Iysine sequence, and the engineered secondary structure elements were unlikely to interfere with the MMP-1 binding to, and hence cleavage of, the designed monomeric peptide molecules, we have proceeded with synthesis of each peptide and subsequent structural and MMP-1 degradability characterizations.

\section{Synthesis, Self-assembly and Structure Characterization of the Designed PAs}

The designed peptide molecules were then successfully synthesized and purified. Their mass and purity were confirmed by electrospray ionization mass spectrometry (ESI-MS) and analytical reverse phase high performance liquid chromatography (RP-HPLC), respectively (Fig. S2-S7+). Critical aggregation concentration (CAC) of PAs is the concentration above which their self-assembly is triggered. To probe the transition of PA monomers into assembled structures, Nile Red assay was performed firstly. Nile Red is a hydrophobic dye with high affinity to the hydrophobic aggregation pocket. ${ }^{45}$ In hydrophilic environment, it has a weak fluorescence, while in hydrophobic environment, it exhibits an increased fluorescence and a pronounced blue shift. ${ }^{45}$ When mixed with various concentrations of PA solutions, Nile Red tends to partition into the hydrophobic core once any PA assemblies appear. Thus, the CAC of PAs can be determined at the point where there is a sharp increase in the fluorescence intensity and a blue shift in the emission peak of Nile Red. ${ }^{46}$ Knowing the CAC values for each PA and PS, the secondary structure of both monomers (< CAC) and assemblies (> CAC) was analysed by circular dichroism (CD) spectroscopy. In addition, the morphology of the assemblies was visualized under TEM. $\beta$-sheet content and stability of the assemblies against dilution were also examined by thioflavin T (ThT) assay and Nile Red assay, respectively.

Even through lacking the hydrophobic alkyl tail and secondary structure promoting peptide segment, PS exhibited a relatively weak self-assembly ability with a high CAC determined at $2 \mathrm{mM}$ (Fig. S8A ${ }^{\dagger}$ ). Accordingly, TEM images of PS at $5 \mathrm{mM}$ and $\mathrm{pH} 7$ showed short fibrous nanostructures (Fig. 2). The self-assembly behaviour of PS at these conditions is largely attributed to its amphipathic structure, with the MMP-1 peptide substrate being relatively hydrophobic compared to the positively charged lysine domain at $\mathrm{pH}$ 7. However, $\mathrm{CD}$ analysis revealed a random coil conformation at $\mathrm{pH} 7$, which was maintained at $\mathrm{pH} 10$ despite the lysine residues being mostly deprotonated (neutral) at this $\mathrm{pH}$. In addition, the short self-assembled fibres observed for PS were not stable enough to resist the effect of dilution (Fig. $3 B$ ). This was further confirmed by the $C D$ spectrum of the diluted aggregates displaying a random coil conformation at $\mathrm{pH}$ 7, similar to the monomer state (Fig. 2).

With the addition of the hydrophobic alkyl tail, PA1 was observed to form relatively longer but sparse nanofibers at $\mathrm{pH}$ 7 (Fig. 2) once above its CAC ( $2 \mathrm{mM}$, Fig. S8Bt). At pH 7, the hydrophobic collapse of the alkyl tails is hindered by the 
repulsive electrostatic forces derived from the positively charged lysine headgroup, preventing the formation of $\beta$-sheet structures below the CAC and the growth of PA1 nanofibers. However, at $\mathrm{pH} 10$, with the lysine-rich domain at one end of the PA1 molecule being mostly deprotonated, a $\beta$-sheet secondary structure was observed for the PA1 monomers and long and intertwined nanofibers were detected under TEM (Fig. 2). Dilution of PA1 solutions prepared above the $\mathrm{CAC}$ at $\mathrm{pH} 7$ showed the presence of aggregated structures (Fig. 3A), indicating that once formed, the PA1 nanofibers are stable against dilution. In fact, a $\beta$-sheet secondary structure was also observed for the diluted PA1 aggregates, instead of the random coil conformation displayed by its monomers at pH 7 (Fig. 2), suggesting stronger internal cohesion in PA1 nanofibers compared to the ones formed by PS. Similar behaviour was observed for another PA system. ${ }^{47}$

Similar to PA1, PA2 also self-assembled into nanofibers despite also presenting a random coil conformation at $\mathrm{pH} 7$ (Fig. 2). However, the PA2 nanofibers were longer and denser compared to the ones formed by PA1 and its CAC was relatively lower $(0.04 \mathrm{mM}$, Fig. $\mathrm{S} 8 \mathrm{C}+$ ). The enhanced self-assembly ability of PA2 may be explained by the introduction of the hydrophobic $\alpha$-helix promoting sequence AAAAAA, which strengths the hydrophobic force among the PA2 molecules for competing with the electrostatic repulsive forces of the charged domain. Even though high density of nanofibers were observed at $\mathrm{pH} 10$, a combination of $\alpha$-helix and $\beta$-sheet secondary structures was detected in the CD spectrum (Fig. 2). Using an online secondary structure analysis tool, BeStSel, ${ }^{48,} 49$ for the PA2 monomers, revealed $20 \%$ of helical content. It indicates that the $\alpha$-helix promoting peptide segment endows PA2 with a slight trend to adopt helical secondary structure. The existence of mixed secondary structures in PAs has been reported before. ${ }^{26}$ Furthermore, unlike PA1, PA2 nanofibers were not stable against dilution (Fig. $3 \mathrm{~B}$ ) and its diluted aggregates displayed a predominant random coil conformation (Fig. 2), which suggests, to some extent, the instability of PA2 nanofibers. The slightly twisted helical conformation of PA2 may lead to a less packed and disordered fibres.

Comprising the well-known $\beta$-sheet forming peptide segment (VVVAAA) used in PA molecules, PA3 exhibited a comparatively low CAC of $0.004 \mathrm{mM}$ (Fig. S8D+), demonstrating significant selfassembly ability. Short nanofibers were observed to form in high density (Fig. 2), which is consistent with what we have reported in our previous study. ${ }^{15}$ As expected, due to the presence of the $\beta$-sheet forming peptide segment, PA3 monomers adopted a defined $\beta$-sheet secondary structure at $\mathrm{pH} 7$, which was enhanced at $\mathrm{pH} 10$. In addition, the short nanofibers were observed to resist to the dilution effect (Fig. $3 A$ ). CD data for diluted PA3 aggregates (Fig. 2) also confirms the persistence of $\beta$-sheet conformation upon dilution with the positive band being more intense compared to PA3 monomers, despite being measured at the same concentration. The higher intensity of the $\beta$-sheet signal is an indicator of increased $\beta$ sheet content once stable assemblies are formed. These results are supported by the study from the Stupp laboratory, in which site-directed spin labelling and electron paramagnetic

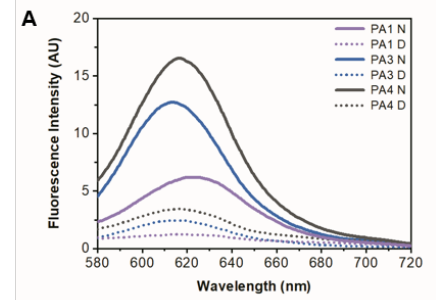

\begin{tabular}{|l|l|l|}
\hline \multicolumn{3}{|c|}{ Maximum Fluorescence Emission } \\
\hline$\#$ & Wavelength & Intensity \\
\hline PA1 nanofibers & 620.0 & 6.1876 \\
\hline PA1 dilution & 619.5 & 1.2327 \\
\hline PA3 nanofibers & 613.5 & 12.6941 \\
\hline PA3 dilution & 614.5 & 2.4450 \\
\hline PA4 nanofibers & 616.5 & 16.5415 \\
\hline PA4 dilution & 615.5 & 3.4432 \\
\hline
\end{tabular}

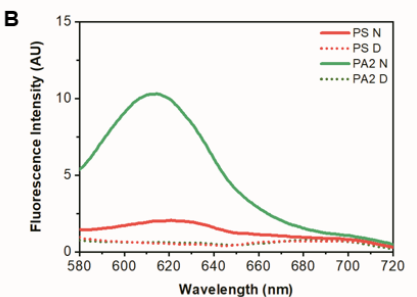

\begin{tabular}{|l|l|l|}
\hline \multicolumn{3}{|c|}{ Maximum Fluorescence Emission } \\
\hline$\#$ & Wavelength & Intensity \\
\hline PS nanofibers & 621.0 & 2.0463 \\
\hline PS dilution & 683.0 & 0.6934 \\
\hline PA2 nanofibers & 614.5 & 10.2807 \\
\hline PA2 dilution & 681.0 & 0.7796 \\
\hline
\end{tabular}

Fig. 3 Stability examination of PA and PS nanofibers: the maximum fluorescence emission wavelength of Nile Red remains for PA1, PA3 and PA4 nanofibers (A) and red-shifted for $P A 2$ and PS nanofibers (B) upon dilution, indicating stable

resonance spectroscopy were used to probe the internal dynamics of PA nanofibers. ${ }^{50}$ It was found that PA molecules assembled within strong $\beta$-sheet nanofibers have slow dynamics and minimal structural instability as a result of the hydrogen bonding network that immobilizes the molecules into a stable supramolecular configuration. ${ }^{50}$ In PA4, in which the positions of the $\beta$-sheet forming peptide segment and MMP-1 peptide substrate were switched compared to PA3, its structure properties and self-assembly behaviour were observed to change. More specifically, an increased CAC $(0.06 \mathrm{mM}$, Fig. $\mathrm{S} 8 \mathrm{E}+$ ) was needed to drive the self-assembly of PA4 into long and straight nanofibers (Fig. 2). These nanofibers were stable against dilution (Fig. 3A) and exhibited a $\beta$-sheet conformation once assembled (Fig. 2). It is likely that by placing the $\beta$-sheet forming peptide segment near to the charged lysine domain, the electrostatic repulsive force of the lysine domain is compromised by the hydrogen bonding of the $\beta$-sheet forming peptide segment, resulting in the reduction of structural curvature for the PA4 assemblies and hence the formation of long and straight nanofibers. However, since the MMP-1 substrate peptide domain was placed closer to the hydrophobic alkyl tail, the rigid structure of the proline and the steric hindrance from the tryptophan are likely to prevent hydrogen bond formation between the amide backbones, which are at the core of the PA4 molecules and necessary for the $\beta$-sheet stabilization. As a result, a random coil conformation was observed for the PA4 monomer at $\mathrm{pH} 7$ (Fig. 2). These results are in agreement with earlier studies, which demonstrated that sequence modifications at the alkyl tail and peptide headgroup interface of PAs could strongly influence the hydrogen bonding and hence their nanostructural morphology. ${ }^{26}$

To further confirm the secondary structure of the selfassembled PA nanofibers provided by CD analysis, thioflavin-T (ThT) assay was performed to examine the presence of $\beta$-sheets in the assemblies. ${ }^{51}$ ThT is a fluorescence dye that increases its emission fluorescence when binding to $\beta$-sheet rich structures.52 As shown in Fig. 2, significant fluorescence enhancement was observed in the PA3 and PA4 nanofibers, 
confirming their dominant $\beta$-sheet content. In contrast, the fluorescence in the PS nanofibers was just slightly higher than that of the blank ThT solution, indicating the absence of $\beta$ sheets in these nanofibers. PA1 nanofibers largely increased the ThT fluorescence, while only minor increase was observed for the PA2 nanofibers, implying less $\beta$-sheet content as a result of the combined conformation within the PA2 assemblies. These observations are in agreement with the $C D$ results.

In summary, the designed PS and PAs could all self-assemble into fibrous nanostructures at $\mathrm{pH} 7$ once above their corresponding CACs. However, only the PA1, PA3 and PA4 nanofibers possess pronounced $\beta$-sheet secondary structure. The short fibrous nanostructures formed by PS display an unstable random coil conformation, while the long nanofibers of PA2 possess combined secondary structures. The designed PS and PAs, with similar peptide sequence but distinct structure properties and self-assembly behaviours (Fig. 2 \& 3), were further utilized as probes to investigate the effect of supramolecular cohesion within their self-assembled nanofibers on MMP-1 degradation efficiency.

\section{Degradation of PA Nanofibers by MMP-1}

To allow direct comparison, all peptide samples (PS and PAs) were prepared at same concentration of $0.5 \mathrm{mM}$ before being incubated with $20 \mathrm{nM}$ active MMP-1 at $37{ }^{\circ} \mathrm{C}$. At this concentration, PS and PA1 should be in their monomeric state, while PA2, PA3 and PA4 should be assembled in the form of nanofibers, according to their CAC values (Table 1). Additionally, PS and PA1 solutions were also prepared at $5 \mathrm{mM}$ to ensure the formation of nanofibers. The MMP-1 degradation activity was then monitored overtime by RP-HPLC. Two peptide fragments are expected as result of MMP-1 cleavage of all PAs and PS. We have selected the expected $C$-terminal peptide fragment (PF, $\mathrm{NH}_{2}$-IWGQKKK-CONH${ }_{2}$ ) from PS, PA1, PA2 and PA3 MMP-1 hydrolysis to monitor degradation. PF was synthesized and could be easily detected in the HPLC trace at around $13 \mathrm{~min}$ (Fig. $\mathrm{S}_{\mathrm{B}}+$ ). Using different $\mathrm{PF}$ concentrations, a calibration curve was constructed (Fig. S12t) and used to quantify the degradation efficiency.

Effect of Hydrophobic Alkyl Tail. As shown in Fig. 4, evident changes were observed in the RP-HPLC spectrum of PS monomer sample after being incubated with active MMP-1 for $24 \mathrm{~h}$, with one peak around $15 \mathrm{~min}$ decreasing (indicated with red arrow), while another peak at approximately $13 \mathrm{~min}$ increasing (indicated with green arrow). The increasing peak was collected and analysed by ESI-MS, and the detected mass corresponds to the mass of PF, the expected $C$-terminal peptide fragment resulting from MMP-1 degradation (Fig. S9+). For the sample containing the PA1 monomers, the increasing peak with shorter elution time appearing around $13 \mathrm{~min}$ was also observed (Fig. 4). However, the intensity of the peak was not as high as that of the PS monomers. As shown in Fig. 4, nearly $95.00 \%$ of PS monomers were degraded by MMP-1 after $24 \mathrm{~h}$, while only $16.33 \%$ degradation was achieved for PA1 monomers. These results indicate that conjugating a
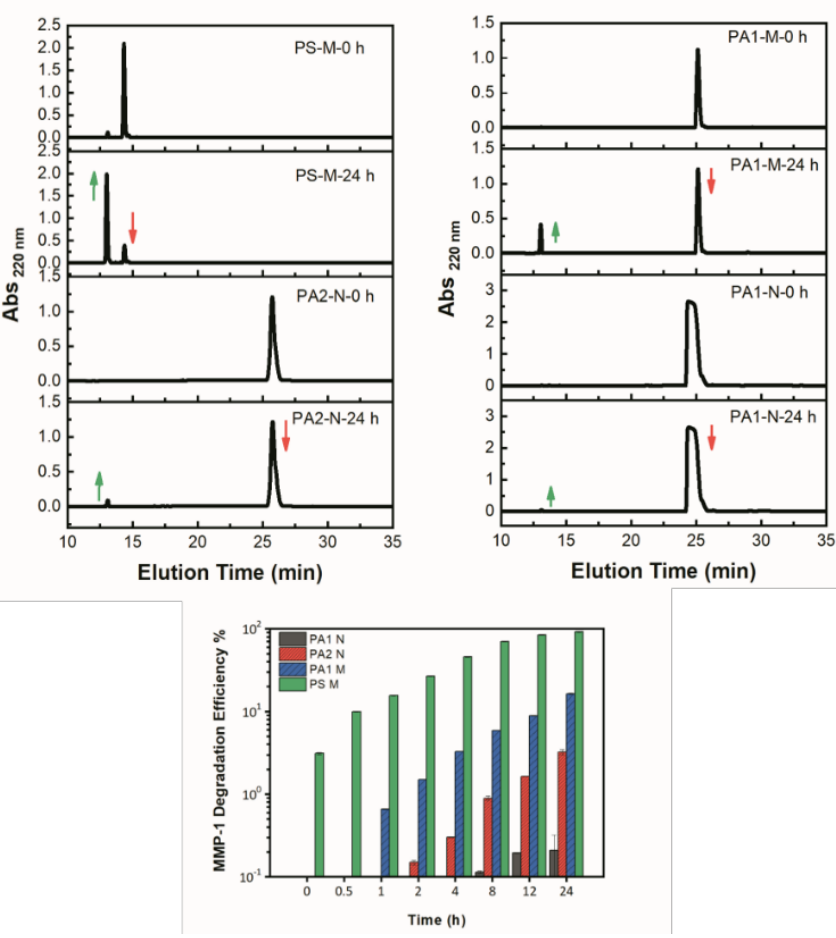

Fig. 4 MMP-1 degradation of PS and PA1 monomers, as well as PA1 and PA2 nanofibers: analytical RP-HPLC spectra (selected elution time range) of $0.5 \mathrm{mM}$ PS, PA1, PA2 and 5 mM PA1 before and after incubation with $20 \mathrm{nM}$ active MMP-1 in TCNB buffer ( $\mathrm{pH}$ 7) at $37^{\circ} \mathrm{C}$ for $24 \mathrm{~h}$; calculated MMP-1 degradation efficiency of both PS and PA1 monomers, as well as PA1 and PA2 nanofibers, measured as
cleavage percentage.

hydrophobic alkyl tail to the $N$-terminus of the MMP-1 cleavable peptide segment could decrease, to some extent, its MMP-1 degradability. Even though the in silico modelling results indicate that productive MMP-1:peptide complexes are possible in each case, i.e. they are not prevented by steric hindrance between the enzyme and structural moieties flanking the central GPQG $\downarrow$ IWGQ peptide, it is also possible that other interactions may interfere with peptide binding or active site availability. For example, given the hydrophobic nature of the palmitic acid chain, it is possible that it may interfere with productive substrate binding by partially occupying the S3 or S1' pockets which are themselves non-polar in nature; thus it acts as a competitive inhibitor. Alternatively, the palmitic acid group may also interact with the enzyme outside of the active site, perhaps subtly changing the way the substrate lies in the catalytic cleft, and therefore reducing the turnover rate.

Effect of Self-assembly. Upon self-assembly, the MMP-1 degradability of PA1 nanofibers ( $5 \mathrm{mM}$ ) was shown to be even more decreased $(0.21 \%)$ compared with the PA1 monomers $(0.5 \mathrm{mM})$ (Fig. 4). To assess if the substrate concentration was a limiting factor for the reduced degradation efficiency, the degradation of PS nanofibers was also examined at $5 \mathrm{mM}$ (Fig. $\left.\mathrm{S} 10^{+}\right)$. The results indicate that the amount of MMP-1 used is still sufficient for degrading a significant amount of PS, confirming that the lower degradation rate of PA1 nanofibers is due to reduced availability of PA1 monomers for enzyme cleavage and not solely to concentration effects. Self-assembly 
induced protease resistance has already been reported in previous studies and explained by the inaccessibility of the protease cleavable domain in the self-assembled nanostructures for the effective protease cleavage. ${ }^{53}$

Effect of Secondary Structure. By comparing the MMP-1 degradability of all self-assembled nanofibers, from PA1 to PA4, the MMP-1 degradability of PA nanofibers was found to be secondary structure dependent. According to our observations, the $\beta$-sheet nanofibers assembled from PA3 and PA4, both containing the well-known $\beta$-sheet forming domain, exhibited extreme resistance to MMP-1 degradation as no peptide fragments were detected within $72 \mathrm{~h}$ (Fig. S11†). Similarly, the MMP-1 degradation could be barely detected for the $\beta$-sheet nanofibers formed by PA1 within $24 \mathrm{~h}$ (Fig. 4), even though it lacks the $\beta$-sheet forming domain and adopted a random coil conformation below the CAC. In contrast, PA2, with $20 \%$ helical content, displayed a slightly increased degradability by MMP-1 after $24 \mathrm{~h}$ (3.22\%, Fig. 4). A possible explanation could be degree of the internal order within the different nanofibers. The relatively loose internal packing of the PA2 nanofibers, indicated by the change in their secondary structure induced by dilution (Fig. 2), suggests their dynamic behaviour and the dissociation of individual PA2 monomers from the nanofiber. In this scenario, MMP-1 is able to cleave the dissociated monomers, shifting the equilibrium in favour of the monomers. Consecutive hydrolysis of free peptide monomers leads to defects in the fibre packing and integrity. The weaker supramolecular cohesion in nanofibers self-assembled from AAAAAA containing PAs was also reported in previous study, confirmed by the exchange of PA molecules between fibres observed by stochastic optical reconstruction microscopy. ${ }^{54}$ The $\beta$-sheet PA3 and PA4 nanofibers are more stable, requiring more time to observe an equilibrium (Fig. S11 ${ }^{\dagger}$ ). Collagenases, such as MMP-1, are generally known for degrading interstitial collagens (Coll I, II and III). However, they can also break down some non-triple helical substrates although with lower efficiency. ${ }^{34}$ Fibrillar collagen is composed of associated individual collagen molecules consisting of three $\alpha$ chains in a helical conformation. ${ }^{55,56}$ Each triple helix measures $300 \mathrm{~nm}$ in length and $1.5 \mathrm{~nm}$ in diameter. ${ }^{34}$ Studies on the crystal structure of MMP-1 revealed a substrate binding site with the shape of a groove $5 \AA$ id wide, indicating that the dimensions of triple-helical collagen are too large to fit in the MMP-1 substrate binding site. ${ }^{34}$ It is thus unlikely that the PA nanofibers would fit into the MMP-1 active site and forming a stable enzyme:substrate complex, as the peptide sequence surrounding the scissile bond must form backbone hydrogen bonds with the outer strand of the catalytic domain's $\beta$-sheet. These interactions cannot take place if this peptide sequence is involved in intermolecular interactions with other peptides, and/or if steric hindrance from neighbouring peptides in the nanofiber prevents a close approach of the enzyme. Work performed by Gregg Fields and collaborators has shed light into the mechanisms by which MMPs cleave native collagen. ${ }^{36}$ Instrumental in these studies were the use of model substrates (triple helical peptides containing potential cleavage sites) and MMP mutants. ${ }^{33}$ It was demonstrated that MMP-1 is able to unfold the triple helix of collagen and cleave the strands of the triple helix. ${ }^{34}$ However, the unfolding capacity of MMP-1 may not been applicable to the $\beta$-sheet conformations, which are maintained through intermolecular hydrogen bonding, instead of the intramolecular hydrogen bonds in helical structures.

\section{Experimental Section}

\section{In Silico Modelling of MMP-1:Peptide Binding}

Models of the binary pre-hydrolysis (Michaelis) complexes formed between MMP-1 and monomeric peptides were generated using the program CNS using a standard $x$-ray crystallography force-filed supplemented with bespoke topology and parameter description for the $\mathrm{N}$-terminal palmitic acid adduct, and employing cartesian molecular dynamicsbased simulated annealing protocols with conjugates-gradient minimisation. ${ }^{57}$ The non-bonded "switching" option was employed in order to smoothly truncate the attractive component of the van der Waals potential to zero at an interatomic distance of between 6.5 and $7.5 \AA$ A. Inter-atomic $(\mathrm{C} \alpha-\mathrm{C} \alpha)$ distance restraints and dihedral angle restraints were employed to maintain the approximate tertiary structure of MMP enzyme domains.

An initial model of MMP-1 in complex with the hexapeptide PQGIWG was generated from the $x$-ray coordinates of the MMP-8 catalytic domain (MMP-8DHPX) in complex with the synthetic inhibitors Pro-Leu-Gly-hydroxamate ${ }^{42}$ and 3mercapto-2-benzylpropanoyl-Ala-Gly- $\mathrm{NH}_{2}{ }^{41}$ (PDB accession codes $1 \mathrm{JAN}$ and $1 \mathrm{JAO}$, respectively). Initially, the hydroxamate and mercaptomethylene moieties were deleted, and the bridging peptide bond was introduced, whilst harmonically restraining the remaining heavy atoms, to generate a MMP$8 \triangle \mathrm{HPX}: \mathrm{PLG} \downarrow$ FAG complex, where $\downarrow$ indicates the scissile bond. The desired MMP-1:PQG $\downarrow$ IWG complex structure was then generated by superposition with the crystal structure of MMP1 (accession code $2 \mathrm{CLT}),{ }^{44}$ mutation of three side chains (L[P2]Q, $\mathrm{F}\left[\mathrm{P} 1^{\prime}\right] \mathrm{I}$ and $\left.\mathrm{A}\left[\mathrm{P2} 2^{\prime}\right] \mathrm{W}\right)$, and simulated annealing of the resultant conformation. In subsequent calculations, the conformation of this PQG $\downarrow$ IWG hexapeptide within the MMP-1 active site was restrained with intramolecular dihedral angle restraints and intermolecular distance restraints. Outside of this region, secondary structure elements within the PAs were imparted with typical $\phi$ and $\psi$ dihedral (torsion) angle restraints. In addition, $\alpha$ helical regions were further stabilised by interatomic distance restraints typical of $(i, i+4)$ hydrogen bonds. No torsional restraints were placed on the tri-lysine and palmitic acid moieties, so their conformations were determined largely by steric repulsion.

All images regarding the in silico modelling were produced using the program PyMol. ${ }^{58}$

\section{Peptide Synthesis and Purification}

All the peptides used in this study were synthesized in a Liberty Blue automated microwave peptide synthesizer (CEM) using the standard fluorenylmethyloxycarbonyl (Fmoc)-based solid phase 
peptide synthesis method. Briefly, 4-methylbenzhydrylamine (MBHA) rink amide resin was used as the solid support to start each synthesis. Amino acid coupling cycles were performed with a mixture of Fmoc-amino acid / 1-hydroxybenzotriazole (HOBt) / N, N'-diisopropylcarbodiimide (DIC) at a molar ratio of $4: 4: 4$, relative to the resin. Fmoc deprotections were performed with $20 \%(\mathrm{v} / \mathrm{v})$ piperidine in dimethylformamide (DMF) for 10 min twice. Palmitic acid $\left(\mathrm{C}_{16} \mathrm{H}_{32} \mathrm{O}_{2}\right)$ was manually coupled to the peptide $\mathrm{N}$-terminus amine for obtaining PA molecules. Coupling was performed overnight under the same conditions as for the Fmoc-amino acids while acetylation of the $N$-terminus was performed for the PS by shaking the peptide-bound resin with acetic anhydride (10\% in DMF) for 20 minutes twice. The final coupling reaction was monitored with Kaiser test to confirm the lack of free amines. PAs and PS were then cleaved from the resin with a mixture of trifluoroacetic acid (TFA) / triisopropylsilane (TIS)/ $\mathrm{H} 2 \mathrm{O}$ at a volume ratio of 95:2.5:2.5 for $2 \mathrm{~h}$ with simultaneous removal of all the protecting groups. The cleavage solution was then collected and the excess TFA removed by rotary evaporation. Cold diethyl ether was added to precipitate the peptide product, which was then collected, washed again with cold diethyl ether and dried under vacuum overnight. Mass of the crude product was analysed via electrospray ionization mass spectrometry (ESI-MS, Agilent).

Peptide purification was performed in an AutoPurification System (Waters) using a preparative reverse-phase (RP) C18 column (XBridge, $130 \AA$, $5 \mu \mathrm{m}, 30 \times 150 \mathrm{~mm}$, Waters) and a gradient of $\mathrm{H} 2 \mathrm{O} /$ Acetonitrile (ACN) (0.1\% TFA) as mobile phase. Fractions containing the PAs were automatically collected when the exact mass signals were detected by a single quadrupole mass detector (SQ Detector 2, Waters). The collected PA fractions were concentrated first by rotary evaporation, then lyophilized and stored at $-20^{\circ} \mathrm{C}$ until further use.

Mass confirmation and purity of all peptides were analysed (1 $\mathrm{mg} / \mathrm{mL}$ ) via ESI-MS and by high-performance liquid chromatography (HPLC, Alliance, Waters), equipped with an analytical RP C18 column (XBridge, $130 \AA$, $3.5 \mu \mathrm{m}, 4.6 \times 150 \mathrm{~mm}$, Waters) and a UV detector set at $220 \mathrm{~nm}$.

\section{Critical Aggregation Concentration (CAC)}

The CAC of PAs was determined according to a previous reported protocol. ${ }^{46}$ Briefly, a hydrophobic solvatochromic dye, Nile Red, was dissolved in acetone and aliquoted to Eppendorf tubes before being left in a dark place to generate a dry film at room temperature. Various concentrations of PA solutions, ranging from $100 \mathrm{~nm}$ to $10 \mathrm{mM}$, were then prepared and added to the Eppendorf tubes to dissolve the Nile Red dry film to a final concentration of $1 \mu \mathrm{M}$. After being aged overnight, each solution was analysed on a LS55 fluorescence spectrometer (PerkinElmer) with a fixed excitation wavelength of $550 \mathrm{~nm}$. Fluorescence emission spectrum with a range from $580 \mathrm{~nm}$ to $720 \mathrm{~nm}$ was then obtained for each solution and its maximum intensity and corresponding wavelength were determined and plotted as function of the logarithm PA concentration. The CAC of each PA was determined at the point where there is a sharp increase in the fluorescence intensity and a blue-shift of the emission maximum.

\section{Stability Test}

The stability of the PA assemblies against dilution was tested using the Nile Red assay. PA solutions were prepared by dissolving the PAs above their CACs firstly. After being incubated with the dry Nile Red film overnight, the fluorescence emission spectrum of each solution was recorded on a LS55 fluorescence spectrometer as described above. A 10-fold dilution of each solution was then prepared in Milli-Q water and its fluorescence emission spectrum was recorded immediately. The red-shift in the emission maximum of diluted sample $<$ CAC) is considered as occurrence of the disassembly process, indicating the instability of the PA assemblies.

\section{Circular Dichroism (CD)}

The secondary structure of PAs was characterized in a Chirascan CD spectrometer (Applied Photophysics) in the far UV region $(190 \mathrm{~nm}-280 \mathrm{~nm})$ at $25 \mathrm{oC}$. Samples were prepared both in the monomer state by directly dissolving the PA below its CAC, and in the self-assembled state by dissolving the PA above its CAC first, followed by dilution before measurement. Each prepared solution was loaded into a $1 \mathrm{~mm}$ path length quartz cuvette and its recorded signal in ellipticity (mdeg) converted to mean residue ellipticity ( $\mathrm{deg} \cdot \mathrm{cm}^{2} \cdot \mathrm{dmol}^{-1}$ ) by the following equation:

$[\theta]=\frac{\theta}{10 \cdot c \cdot l \cdot n}$

where $\theta$ is the recorded ellipticity in mdeg, $c$ is the PA concentration in $M, l$ is the light path length of the cuvette in $\mathrm{cm}$, and $n$ is the number of amino acid residues in the peptide molecules.

\section{Thioflavin T (ThT) Assay}

PA solutions were prepared by dissolving the PAs above their CACs first, followed by dilution in Milli-Q water to a concentration of $0.1 \mathrm{mM}(\mathrm{pH} 7.0)$, before being mixed with an identical volume of ThT aqueous solution with a concentration of $50 \mu \mathrm{M}$. After being incubated for $1 \mathrm{~h}$, the fluorescence emission spectrum of each sample was recorded on the LS55 fluorescence spectrometer from $460 \mathrm{~nm}$ to $660 \mathrm{~nm}$, with the excitation wavelength fixed at $440 \mathrm{~nm}$.

\section{Transmission Electron Microscopy (TEM)}

Samples for TEM imaging were prepared by casting $10 \mu \mathrm{L}$ PA solution ( $5 \mathrm{mM}$ for PS and PA1 and $0.5 \mathrm{mM}$ for PA2, PA3 and PA4) on a carbon film-coated copper grid with 400 square mesh (Agar Scientific). Excess PA solution on the grid was removed with a piece of filter paper. The samples were then negatively stained by loading $10 \mu \mathrm{L} 2 \%(\mathrm{v} / \mathrm{v}$ ) uranyl acetate solution (Agar Scientific) on the grid and the excess staining solution removed after $30 \mathrm{~s}$. Samples were allowed to dry at room temperature 
for at least $3 \mathrm{~h}$ before being examined on a JEOL-1230 TEM with an acceleration voltage of $80 \mathrm{kV}$. TEM images were recorded with a SIS Megaview III wide angle CCD camera.

\section{MMP-1 Cleavage of Designed Peptides}

$40 \mu \mathrm{L}$ of $80 \mu \mathrm{g} / \mathrm{mL}$ poMMP-1 (human rheumatoid synovial fibroblast proenzyme; Merck Millipore; catalogue number: 444208-5UG; Lot number: 2625650; specific activity: > 100.0 $\mathrm{mU} / \mathrm{mg} \mathrm{P}$ ) was first diluted into $160 \mu \mathrm{L}$ TNCB buffer $(50 \mathrm{mM}$ tris(hydroxymethyl)aminomethane hydrochloride (Tris- $\mathrm{HCl}$ ), $150 \mathrm{mM}$ sodium chloride $(\mathrm{NaCl}), 10 \mathrm{mM}$ calcium chloride $(\mathrm{CaCl} 2)$, and $\left.0.05 \% \mathrm{Brij}^{\circledR} 35, \mathrm{pH} 7\right)$, and then activated by reacting with $20 \mu \mathrm{L}$ of $50 \mu \mathrm{M}$ p-aminophenylmercuric acetate (APMA) dissolved in 0.1 sodium hydroxide $(\mathrm{NaOH})$ at $37^{\circ} \mathrm{C}$ for 3 h. After activation, $30 \mu \mathrm{L}$ of MMP-1 was added to $470 \mu \mathrm{L}$ of each peptide solution and incubated at $37^{\circ} \mathrm{C} .40 \mu \mathrm{L}$ aliquots of the reaction mixture were sampled at different time points $(0,0.5$, $1,2,4,8,12$, and $24 \mathrm{~h}$ ), flash frozen in liquid nitrogen and stored at $-20^{\circ} \mathrm{C}$ until further analysis by RP-HPLC.

The samples were analysed on an Alliance HPLC system (Waters), equipped with an analytical RP C18 column (XBridge, $130 \AA, 5 \mu \mathrm{m}, 30 \times 150 \mathrm{~mm}$, Waters). A mobile phase of $\mathrm{H} 2 \mathrm{O}$ / ACN $(0.1 \%$ TFA) under the gradient of $98 \%$ to $0 \% \mathrm{H} 2 \mathrm{O}$ from 5 $\min$ to $35 \mathrm{~min}$ was utilized. The detection UV wavelength was set at $220 \mathrm{~nm}$. The PF resulting from MMP-1 degradation was collected when significant UV signal appeared and then identified by ESI-MS.

To quantify the degradation rate, a series of PF solutions with different concentrations were prepared and injected into the RP-HPLC. Standard curve of PF was established by plotting the peak area versus concentration. The MMP-1 degradation efficiency for each PA was calculated by integrating the PF peak area in the corresponding RP-HPLC chromatogram and then converting into concentration from the standard curve (Fig. $\mathrm{S} 12+)$. Data is presented as average values from three independent experiments with error bars representing the standard deviation.

\section{Conclusions}

A series of PAs, all comprising the same MMP-1 cleavable domain but with variations in the sequence and location of the secondary structure promoting segment, were utilized to probe the hydrolysis of their assemblies by MMP-1. We found that through rational molecular design, the self-assembly behaviour of PAs could be tuned, leading to variations in their CACs, secondary structures, assembled morphologies, and most notably their susceptibility to MMP-1 degradation. Adding a hydrophobic tail to the MMP-1 cleavable peptide sequence, as well as self-assembly, reduced the MMP-1 degradation efficiency. Additionally, tuneable degradability of the PA nanofibers by MMP-1 could be achieved by tweaking their secondary structures, adjusted primarily through the deletion or alteration of the secondary structure promoting segments in the PA molecules. To the best of our knowledge, this is the first demonstration that the $\beta$-sheet composition in supramolecular
PA nanostructures influences their susceptibility to MMP-1 degradation. We believe our findings provide fundamental insights into the generic design of MMP-1 responsive supramolecular peptide biomaterials, which can be further exploited as ECM mimics, tissue engineering scaffolds, as well as drug delivery vehicles.

\section{Conflicts of interest}

There are no conflicts to declare.

\section{Acknowledgements}

Y. S. and H. A. gratefully acknowledge the Seed Award in Science (210122/Z/18/Z) granted by the Wellcome Trust. D. F. acknowledges the People Programme (Marie Curie Actions) of the European Union's Seventh Framework Programme (FP7/2007-2013) under REA grant agreement no 608765. J. B. acknowledges Marie Sklodowska-Curie Individual Fellowship granted by the European Commission (MSCA-IF-2014-658351).

\section{References}

1. J. Gross and C. M. Lapiere, Proceedings of the National Academy of Sciences of the United States of America, 1962, 48, 1014-1022.

2. C. Bonnans, J. Chou and Z. Werb, Nature reviews. Molecular cell biology, 2014, 15, 786-801.

3. A. Page-McCaw, A. J. Ewald and Z. Werb, Nature reviews. Molecular cell biology, 2007, 8, 221-233.

4. K. Kessenbrock, V. Plaks and Z. Werb, Cell, 2010, 141, 5267.

5. T. Turpeenniemi-Hujanen, Biochimie, 2005, 87, 287-297.

6. X. X. Zhang, H. S. Eden and X. Chen, Journal of controlled release : official journal of the Controlled Release Society, 2012, 159, 2-13.

7. M. Zelzer, S. J. Todd, A. R. Hirst, T. O. McDonald and R. V. Ulijn, Biomaterials science, 2013, 1, 11-39.

8. C. F. Anderson and H. Cui, Industrial \& engineering chemistry research, 2017, 56, 5761-5777.

9. H. Cui, M. J. Webber and S. I. Stupp, Biopolymers, 2010, 94, 1-18.

10. A. Dehsorkhi, V. Castelletto and I. W. Hamley, Journal of peptide science : an official publication of the European Peptide Society, 2014, 20, 453-467.

11. K. Sato, M. P. Hendricks, L. C. Palmer and S. I. Stupp, Chemical Society reviews, 2018, 47, 7539-7551.

12. M. P. Lutolf, J. L. Lauer-Fields, H. G. Schmoekel, A. T. Metters, F. E. Weber, G. B. Fields and J. A. Hubbell, Proceedings of the National Academy of Sciences of the United States of America, 2003, 100, 5413-5418.

13. H. W. Jun, V. Yuwono, S. E. Paramonov and J. D. Hartgerink, Adv Mater, 2005, 17, 2612-+.

14. H. W. Jun, S. E. Paramonov, H. Dong, N. Forraz, C. McGuckin and J. D. Hartgerink, Journal of biomaterials science. Polymer edition, 2008, 19, 665-676.

15. D. S. Ferreira, Y. A. Lin, H. Cui, J. A. Hubbell, R. L. Reis and H. S. Azevedo, Advanced healthcare materials, 2015, 4, 602-612. 
16

J. K. Kim, J. Anderson, H. W. Jun, M. A. Repka and S. Jo, Molecular pharmaceutics, 2009, 6, 978-985.

17. D. Koda, T. Maruyama, N. Minakuchi, K. Nakashima and M. Goto, Chemical communications, 2010, 46, 979-981.

18 A. Tanaka, Y. Fukuoka, Y. Morimoto, T. Honjo, D. Koda, M Goto and T. Maruyama, Journal of the American Chemical Society, 2015, 137, 770-775.

19. Y. Shi, Y. Hu, G. Ochbaum, R. Lin, R. Bitton, H. Cui and H. S. Azevedo, Chemical communications, 2017, 53, 7037-7040. S. Sokic, M. C. Christenson, J. C. Larson, A. A. Appel, E. M. Brey and G. Papavasiliou, Biomaterials science, 2014, 2, 1343-1354.

21. J. Patterson and J. A. Hubbell, Biomaterials, 2010, 31, 78367845.

22. J. Patterson and J. A. Hubbell, Biomaterials, 2011, 32, 13011310.

C. Chen, Y. Zhang, Z. Hou, X. Cui, Y. Zhao and H. Xu, Biomacromolecules, 2017, 18, 3563-3571.

24. M. C. Giano, D. J. Pochan and J. P. Schneider, Biomaterials, 2011, 32, 6471-6477.

$25 . \quad J$. Son, D. Kalafatovic, M. Kumar, B. Yoo, M. A. Cornejo, M. Contel and R. V. Ulijn, ACS nano, 2019, 13, 1555-1562.

26. S. E. Paramonov, H. W. Jun and J. D. Hartgerink, Journal of the American Chemical Society, 2006, 128, 7291-7298.

27. E. T. Pashuck, H. G. Cui and S. I. Stupp, Journal of the American Chemical Society, 2010, 132, 6041-6046.

$28 . \quad$ D. Missirlis, A. Chworos, C. J. Fu, H. A. Khant, D. V. Krogstad and M. Tirrell, Langmuir, 2011, 27, 6163-6170.

29. C. J. Newcomb, S. Sur, J. H. Ortony, O. S. Lee, J. B. Matson, J. Boekhoven, J. M. Yu, G. C. Schatz and S. I. Stupp, Nature communications, 2014, 5, 3321.

30.

H. Nagase, R. Visse and G. Murphy, Cardiovascular research, 2006, 69, 562-573.

31. H. G. Welgus, J. J. Jeffrey and A. Z. Eisen, J Biol Chem, 1981, 256, 9511-9515.

32. H. G. Welgus, J. J. Jeffrey, G. P. Stricklin and A. Z. Eisen, J Biol Chem, 1982, 257, 11534-11539.

S. W. Manka, F. Carafoli, R. Visse, D. Bihan, N. Raynal, R. W. Farndale, G. Murphy, J. J. Enghild, E. Hohenester and H. Nagase, Proceedings of the National Academy of Sciences of the United States of America, 2012, 109, 12461-12466. . Chung, D. Dinakarpandian, N. Yoshida, J. L. Lauer-Fields, G. B. Fields, R. Visse and H. Nagase, The EMBO journal, 2004, 23, 3020-3030.

35. A. Knapinska and G. B. Fields, Chembiochem : a European journal of chemical biology, 2012, 13, 2002-2020.

36. J. L. Lauer-Fields, K. A. Tuzinski, K. Shimokawa, H. Nagase and G. B. Fields, J Biol Chem, 2000, 275, 13282-13290. H. Nagase and G. B. Fields, Peptide Science, 1996, 40, 399416

38. M. Levitt, Biochemistry, 1978, 17, 4277-4285.

39. S. J. Smith, K. Du, R. J. Radford and F. A. Tezcan, Chemical science, 2013, 4, 3740-3747.

40. R. H. Zha, Y. S. Velichko, R. Bitton and S. I. Stupp, Soft Matter, 2016, 12, 1401-1410.

41.

F. Grams, P. Reinemer, J. C. Powers, T. Kleine, M. Pieper, H. Tschesche, R. Huber and W. Bode, European journal of biochemistry, 1995, 228, 830-841.

42 Piper, H. Tschesche and W. Bode, Febs Lett, 1994, 338, 227 233.

U. Eckhard, P. F. Huesgen, O. Schilling, C. L. Bellac, G. S. Butler, J. H. Cox, A. Dufour, V. Goebeler, R. Kappelhoff, U. A. D. Keller, T. Klein, P. F. Lange, G. Marino, C. J. Morrison, A. Prudova, D. Rodriguez, A. E. Starr, Y. Wang and C. M. Overall, Matrix biology : journal of the International Society for Matrix Biology, 2016, 49, 37-60.

44. S. Iyer, R. Visse, H. Nagase and K. R. Acharya, Journal of molecular biology, 2006, 362, 78-88.

45. M. C. A. Stuart, J. C. van de Pas and J. B. F. N. Engberts, J Phys Org Chem, 2005, 18, 929-934.

46. Y. Shi, R. Lin, H. Cui and H. S. Azevedo, Methods in molecular biology, 2018, 1758, 11-26.

47. M. N. Walter, A. Dehsorkhi, I. W. Hamley and C. J. Connon, Biomaterials science, 2016, 4, 346-354.

48. A. Micsonai, F. Wien, L. Kernya, Y. H. Lee, Y. Goto, M. Refregiers and J. Kardos, Proceedings of the National Academy of Sciences of the United States of America, 2015, 112, E3095-3103.

49. A. Micsonai, F. Wien, E. Bulyaki, J. Kun, E. Moussong, Y. H. Lee, Y. Goto, M. Refregiers and J. Kardos, Nucleic acids research, 2018, 46, W315-W322.

50. J. H. Ortony, C. J. Newcomb, J. B. Matson, L. C. Palmer, P. E. Doan, B. M. Hoffman and S. I. Stupp, Nature materials, 2014, 13, 812-816.

51. Y. Li, Y. Z. Wang, S. H. Ou, L. L. Lock, X. K. Xu, S. Ghose, Z. J. Li and H. G. Cui, Biomacromolecules, 2017, 18, 3611-3620. M. Biancalana and S. Koide, Bba-Proteins Proteom, 2010, 1804, 1405-1412.

53. L. L. Lock, A. G. Cheetham, P. Zhang and H. Cui, ACS nano, 2013, 7, 4924-4932.

54. R. M. da Silva, D. van der Zwaag, L. Albertazzi, S. S. Lee, E. W. Meijer and S. I. Stupp, Nature communications, 2016, 7, 11561.

55. J. K. Mouw, G. Ou and V. M. Weaver, Nature reviews. Molecular cell biology, 2014, 15, 771-785.

S. Camarero-Espinosa, B. Rothen-Rutishauser, E. J. Foster and C. Weder, Biomaterials science, 2016, 4, 734-767.

A. T. Brunger, P. D. Adams, G. M. Clore, W. L. DeLano, P. Gros, R. W. Grosse-Kunstleve, J. S. Jiang, J. Kuszewski, M. Nilges, N. S. Pannu, R. J. Read, L. M. Rice, T. Simonson and G. L. Warren, Acta Crystallogr D, 1998, 54, 905-921.

58. W. L. DeLano, http://www. pymol. org, 2002. 